\title{
Improved Ant Colony Algorithm for Optimal Solution TSP
}

\author{
Tianyuan Zhou \\ School of computer engineering, Huaihai Institute of Technology, Lianyungang, Jiangsu, China \\ tyhope2999@163.com
}

Keywords: Ant Colony Algorithm; Distance; Pheromone; Iteration

\begin{abstract}
Based on the ant colony algorithm analysis and research, this paper proposed an improved ant colony algorithm. Through updating pheromone and optimal search strategy, then applied to the Traveling Salesman Problem (TSP), effectively improved the searching capability of the algorithm. Finally through the simulation testing and analysis, verified that the improved ant colony algorithm is effective, and has good performance.
\end{abstract}

\section{Introduction}

Traveling salesman problem (TSP) is a typical NP problem, which is assumed to have $N$ cities, a traveling salesman goes from one city, accesses to all cities only once, finally returns to the starting city, and then finds a shortest tour path [1,2,3]. The ACA was a new simulated evolutionary algorithm, which simulates the natural ants foraging process of the group, ants release pheromone on the path when foraging process, then ants select the transfer path based on the concentration of pheromones, eventually all ants will take the shortest path from the nest to the food [4].

Although ant colony algorithm has strong robustness, the convergence restricts the algorithm application. Many researchers have put forward the improved ant colony algorithm, such as minimum spanning tree ant colony, cellular ant algorithm [5]. Although the performance of the algorithm can be improved, it is more complex to implement. Based on the analysis of ant colony algorithm research, this paper proposed improvements to the pheromone updating and searching strategy, and improved the optimal solution for solving traveling salesman problem.

\section{Basic principle of ant colony algorithm to solve TSP problems}

Through research, biologists find ants foraging is a complex group behavior. In search of food source, ants will release a pheromone on its way, and also can percept pheromones released by other ants [6]. Ants release more pheromone amount on the shorter path, also the pheromone concentration of the path increases gradually, and the numbers of ants choose this path is more and more. Ultimately, the entire ants concentrate to the shortest path in the role of the positive feedback, and then this is the optimal solution of optimization problems [7].

This paper assumes the number of ants of the entire ant colonies is $M$, and the number of cities is $N$, the distance between city $i$ and city $j$ is $d_{i j}(i, j=1,2, \cdots, N), \tau_{i j}(t)$ is the pheromone concentration of the path between city $i$ and city $j$ at the time $\tau$. At the initial moment, the same pheromone concentration on the connection path between the each cities, defines as $\tau_{i j}(0)=\tau_{0}$.

This paper assumes the ant $k(k=1,2, \cdots, M)$ determines to access the next city by pheromone concentration of the various cities connection path, and defines $P_{i j}^{k}(t)$ as probability of ant from city $i$ to city $j$ at time $\tau$, formula shown on the Eq.1 [8, 9].

$$
P_{i j}^{k}= \begin{cases}\frac{\left[\tau_{i j}(t)\right]^{\alpha} \cdot\left[\eta_{i j}(t)\right]^{\beta}}{\sum_{s \in \text { allow }_{k}}\left[\left[\tau_{i s}(t)\right]^{\alpha} \cdot\left[\eta_{i s}(t)\right]^{\beta}\right]} & s \in \text { allow }_{k} \\ 0 & s \notin \text { allow }_{k}\end{cases}
$$

In the Eq.1, $\eta_{i j}(t)$ is heuristic function, $\eta_{i j}(t)=1 / d_{i j}$ is expectation of ant from city $i$ to city $j$. allow $_{k}$ is the ant $k$ to be accessed set, at the beginning, there are $(N-1)$ elements in 
allow $_{k}$, along with the time advancement, the element decreases ceaselessly in allow ${ }_{k}$ until it is empty, it means that access of all cities is completed. $\alpha$ is pheromone important degree factor, the higher of its value, the greater role of the heuristic function in the transfer, the ants will be transferred to the short distance of the city with high probability.

When the ants release pheromone, at the same time, pheromone will be gradually disappearing on the connection path between various cities. Therefore, when all ants complete one cycle, the pheromone concentration on the connection path between various cities need real-time updates, set $\rho(0<\rho<1)$ as parameters of the degree of pheromone evaporation factor, such as Eq.2 shows.

$$
\begin{cases}\tau_{i j}(t+1)=(1-\rho) \tau_{i j}(t)+\Delta \tau_{i j} & \\ \Delta \tau_{i j}=\sum_{k=1}^{n} \Delta \tau_{i j}^{k} & 0<\rho<1\end{cases}
$$

Which $\Delta \tau_{i j}^{k}$ represents the $k$-ant release the pheromone concentration on the connection path from city $i$ to city $j, \Delta \tau_{i j}$ represents sum of all ants release pheromone concentration on the connection path from city $i$ to city $j$.

\section{Improved algorithm and Application}

\section{Improved Algorithm}

The study found that in the search for the optimal path of the TSP problem, the next city to choose always tended to the recent city [10]. Therefore, this article presented the city data storage structure was expressed with cross chain table, the chain table stored city distance of the nearer cities. The number of cities related to the distance between the adjacent cities, also related to the pheromone on the path. In the search path, Ants searched for the next city from the same row and the same column of the cross chain table of the city, rather than from the rest of cities, so the non-optimal solutions reduced in algorithm solution space, and also the search range. Thus sped up the convergence of the algorithm, improved the performance of the algorithm.

Ants in the search path were based on the amount of pheromone on the path to choose the transfer direction, in order to improve the efficiency of using cross chain table as search strategy; this paper improved the pheromone based on the definition of original pheromone, such as Eq.3.

$$
r=\frac{M-l(k)}{M}
$$

$\mathrm{M}$ is the number of ants, $l(k)$ is the path length of the sort value. The path is shorter, the smaller of $l(k)$ value. So Eq.2 was updated, as Eq.4 and Eq.5 shown, which $\sigma$ is parameter adjustment factor.

$$
\begin{aligned}
& \Delta \tau_{i j}=\rho^{*} \sigma^{*} r / L_{k} \\
& \tau_{i j}(t+n)=\left\{\begin{array}{l}
(1-\rho) \tau_{i j}(t)+\Delta \tau_{i j} \\
0
\end{array}\right.
\end{aligned}
$$

\section{Application Steps}

Firstly, In the calculation of the beginning, this paper need to initialize the relevant parameters, such as the number of ant $M$, pheromone important degree factor $\alpha$, heuristic function important degree factor $\beta$, pheromone volatile factor $\rho$, the total amount of pheromone released $Q$, the maximum number of iterations $i \max$ and initial value of iteration $i=1$.

Secondly, this paper sets every ant in different starting points by random, $x(x=1,2, \cdots, m)$ calculates the next city each ant to visit in accordance with the Eq.1, until all of the ants accesses all cities.

Thirdly, calculate of all the ants' path length $L_{k}$ and path length of the sort value $l(k)$, record the optimal solution in the current number of iterations. Meanwhile, update pheromone concentration on the path between various cities, according to theEq.4 andEq.5. 
Finally, if $i<i \max$, then order $i=i+1$, then empty the table of ant path, go back to step 2; otherwise, terminate computation, and output optimal solution.

\section{Experimental and simulation analysis}

This paper took chn31 in general TSP test library TSPLIB as example [11], compared the improved algorithm with the basic ant colony algorithm. In the test, this paper found the shortest path of ants in each iteration, and appropriately increased the probability values of $\alpha$ to enhance the guiding role of pheromone of the path, correspond reduced the value of $\beta$, to reduce the effect of heuristic information in the probability calculate. After many trials, the test data were shown in table 1.

Table 1. Contrast of shortest distance between basic ACA and improved ACA

\begin{tabular}{|c|c|c|}
\hline Shortest distance & $\begin{array}{c}\text { Basic ACA } \\
\text { Shortest distance }\end{array}$ & $\begin{array}{c}\text { Improved ACA } \\
\text { Shortest distance }\end{array}$ \\
\hline$m=100, \alpha=5, \beta=10, \rho=0.4, Q=1$ & 16296.3142 & 16107.2553 \\
\hline$m=100, \alpha=6, \beta=8, \rho=0.3, Q=1$ & 15894.7306 & 15778.668 \\
\hline$m=50, \alpha=7, \beta=7, \rho=0.2, Q=1$ & 16160.19 & 15965.334 \\
\hline$m=50, \alpha=8, \beta=6, \rho=0.1, Q=1$ & 15973.1864 & 15618.5242 \\
\hline$m=100, \alpha=10, \beta=1, \rho=0.1, Q=10$ & 15706.5249 & 15603.6459 \\
\hline
\end{tabular}



Fig.1 Shortest distance shown by optimal path



Fig.2 Contrast of distance

Table 1 showed that, under the same initial conditions, the improved ant colony algorithm gets the shorter path than the basic ant colony algorithm. At the same time, time is relatively short. Fig. 1 is the shortest optimization distance path of 31-cities. Fig.2 is the contrast of the shortest distance and the average distance. From Fig.2, it could be seen when the iteration number was about 25 times, we got the shortest distance, while starting the iteration process, the average distance had greater shock, and when the number of iterations exceeded 50 times, the average distance had relatively flat and mild. So with the improved algorithm, we could find the optimal solution with fewer number of iterations, effectively avoided the local optimization.

\section{Conclusion}

In this paper, based on the traditional ant colony algorithm, put forward by changing pheromone and using cross chain table as search strategy to improve the ant colony algorithm. With the instance of TSP, simulation experiments were carried out, and compared the experimental results. It showed that the improved algorithm had faster convergence speed, less iteration number and better effect of stability. 


\section{References}

[1] M. Dorigo and L.M. Gambardella: Ant Colonies for the Traveling Sales Man Problem, Biosystems, Vol.43 (1997), p. 73-81

[2] Liping Zhang, Min Yao and Nenggan Zheng: Optimization and Improvement of Genetic Algorithms Solving Traveling Salesman Problem, International Conference on Image Analysis and Signal Processing (IEEE Conference publications, 2009), p. 327-332

[3] F.T. Lin, C.Y. Kao and C.C. Hsu: Applying the Genetic Approach to Simulated Annealing in Solving Some NP-Hard Problems, IEEE Trans. Sys., Men. Cyber. Vol .23 (1993), p. 1167 - 1752.

[4] A. Colomi, M. Dorigo, V. Maniezzo, et al: Distributed Optimization by Ant Colonies. Proc. of the 1st European Conference on Artificial Life (1991), p. 134-142

[5] R.J. Mullen, D. Monekosso, S. Barman and P. Remagnino: A review of Ant Algorithms, Expert Systems with Applications, Vol.36 (2009), p. 9608-9617

[6] S.C. Chu, J.F. Roddick and J.S. Pan: Ant Colony System with Communication Strategies, Sci. Inform Vol.167 (2004), p. 63-76

[7] F.C. Vieira, A.D. Doria Neto and J.A. Costa, An Efficient Approach to the Travelling Salesman Problem using Self-organizing Maps, Internat. J. Neural.Syst. Vol.13 (2003),p. 59-66

[8] Z.P. Yang, L.M. Xin and Y. Wei: On Application of Reformed Ant Colony Algorithm in TSP Problems, Shanxi Architecture, Vol.36 (2010), p. 9-10

[9] C. Voudouris and E. Tsang: Guided Local Search and its Application to the Traveling Salesman Problem. Eur.J. Oper. Res. Vol.113(1999), p. 469-499

[10] H.H. Hoos and T. Stutzle: Stochastic Local Search: Foundations and Applications, Elsevier, Amsterdam, The Netherlands (2004)

[11] Information on http://www.iwr.uni-heidelberg.de/groups/comopt/software/ TSPLIB95/tsp/ 